\title{
REFLEXIÓN SOBRE CUATRO EJES DE INTERSECCIÓN DENTRO DE LA GESTIÓN EN LA EDUCACIÓN EN MÉXICO
}

\author{
Alma Rosa Hernández Mondragón ${ }^{1}$ \\ amhm@ulsa.mx \\ UNIVERSIDAD LA SALLE. MÉXICO \\ Marcela Rosario Salazar Ibáñez ${ }^{2}$ \\ marcela.salazar@ulsa.mx \\ UNIVERSIDAD LA SALLE. MÉXICO \\ Karina Trejo Rodríguez ${ }^{3}$ \\ UNIVERSIDAD AUTÓNOMA METROPOLITANA. MÉXICO
}

Recibido: 8 de marzo de 2018

Aceptado: 8 de mayo de 2018

DOI: http://dx.doi.org/10.15366/didacticas2018.19.001

\section{Resumen}

Desde una perspectiva Docente, se pueden apreciar varios ejes que han impactado a la Gestión desde que ésta se realiza con mayor empeño dentro de la Educación en México, sobre todo en el nivel Básico; desde siempre el liderazgo en primer término, ha marcado la forma de dirigir una Institución Educativa, existen numerosos estudios e investigaciones dando respuesta a un sinnúmero de tipos de liderazgo, así como a la manera de aplicarlo. En segundo término, este ensayo marca la pauta para reflexionar también sobre la Evaluación y Calidad Educativas, prácticamente van de la mano, ya que ambas representan un cambio sustancial que no es fácil implantar dentro de una Institución. El tercer término plantea qué tanto una Política Educativa de un determinado sexenio impacta realmente a la Educación de ese tiempo y se permea al presente; por último, se aprecia la necesidad de crear una Reforma Educativa que realmente funcione en la mayor parte del territorio, concretando con algunas pautas que invitan a la reflexión.

Palabras clave: Gestión; Liderazgo; Evaluación; Calidad Educativa; Política Educativa; Reforma Educativa; Directivos.

\footnotetext{
${ }^{1}$ Investigadora y Docente de Tiempo Completo. Centro Lasallista de Investigación Pedagógica de la Región (CLIPER). Fac. de Humanidades y Ciencias Sociales. Universidad La Salle. México. 5278-9500 ext. 2252.

${ }^{2}$ Investigadora y Docente de Tiempo Completo. Centro Lasallista de Investigación Pedagógica de la Región (CLIPER). Fac. de Humanidades y Ciencias Sociales. Universidad La Salle. México. 5278-9500 ext. 2238.

3 Profesora Investigadora Titular "C" de Tiempo Completo de la Universidad Autónoma Metropolitana, Unidad Cuajimalpa, Docente de asignatura e Integrante del Grupo de Investigación, desarrollo e innovación (GIDi) Gestión liderazgo y calidad en las Instituciones educativas de la Universidad La Salle. México.
} 


\begin{abstract}
From a teaching perspective, we can see several axes that have impacted Management since it is carried out with greater effort in Education in Mexico, especially at the Basic level; always the leadership in the first place, has marked the way of directing an Educational Institution, there are numerous studies and investigations giving answer to a number of types of leadership, as well as to the way to apply it. Secondly, this essay sets the tone to reflect on the Educational Evaluation and Quality, they practically go hand in hand, since both represent a substantial change that is not easy to implement within an Institution. The third term raises how much an Educational Policy of a given sexennial really impacts the Education of that time and permeates the present; Finally, we appreciate the need to create an Educational Reform that really works in most of the territory, specifying with some guidelines that invite reflection.
\end{abstract}

Keywords: Management; Leadership; Evaluation; Educational Quality; Educational Policy; Educational Reform; Directors. 


\section{1.- LIDERAZGO DIRECTIVO}

Debemos de empezar por definir qué es liderazgo, el cual se entiende como el conjunto de habilidades gerenciales o directivas que un individuo tiene para influir en la forma de ser de las personas o en un grupo determinado, haciendo que este equipo trabaje con entusiasmo, en el logro de metas y objetivos.

Ahora bien, el liderazgo directivo a nivel de escuelas juega un rol altamente significativo en el desarrollo de cambios en las prácticas docentes y en el impacto que presentan sobre la calidad del aprendizaje de los alumnos en las Instituciones.

Tienen a su vez, la responsabilidad de ejercer un liderazgo y son quienes impulsan el desarrollo de la Cultura Escolar, apoyando y fomentando una Gestión de Calidad como proceso fundamental para la mejora de procesos que ocurren en un establecimiento escolar (MINEDUC, 2004). Desde una perspectiva tradicional, unidireccional y estática, cabe destacar que, en el desarrollo de estas tareas, el líder se relaciona con procesos de influencia sobre la conducta de los miembros de una organización, ejerciendo su poder de manera informal e influyendo en las metas que el grupo se plantea. En este sentido, "se vale de los vínculos sociales que establece con los otros miembros, sus características personales, su capacidad comunicativa, sus conocimientos sobre la materia u otras destrezas que pone en juego" (López, Sánchez y Murillo, 2003).

Tanto Directivos como profesores, asocian liderazgo, mediante conceptos bastante coincidentes en sus significados. Relacionan, por ejemplo, liderazgo con "director" o "dirigente", como una forma de atribuir importancia o relevancia a las funciones de la persona encargada de encauzar los procesos de "dirigir" una organización y de "influir en la conducta de los miembros de la comunidad educativa con el fin de realizar determinadas acciones que son consecuencia de los objetivos que han fijado en común y que se aceptan como adecuados para la educación" (Antúnez, en López et al.,2003, p.270).

Reflexionando sobre la Gestión Escolar desde una perspectiva global, no se puede dejar de reconocer que la eficiencia del aprendizaje de los alumnos y la efectividad docente se ven afectadas por el liderazgo. Y en la mayoría de las ocasiones el obtener un resultado significativo, es estimulado por un buen reconocimiento y apoyo de toda la comunidad educativa.

Un liderazgo exitoso dentro de las entidades educativas casi siempre se logra en parte por el aprendizaje de los estudiantes que justifica dos afirmaciones (Leithwood, Seashore Louis, Anderson y Wahlstrom, 2004):

1. El liderazgo es el segundo factor intra-escuela, después del trabajo docente en el que más se contribuye al logro de aprendizajes de los alumnos.

2. Los efectos del liderazgo usualmente son mayores en instituciones donde son más necesarios los logros de aprendizajes (Ej. escuelas vulnerables).

El segundo punto no implica que no hay que preocuparse de la calidad del liderazgo en escuelas de altos resultados, que no se encuentran en circunstancias difíciles, sino que 
la visibilidad del impacto de un liderazgo eficaz es mayor en escuelas vulnerables. De hecho, existen instancias documentadas de escuelas de bajos resultados en condiciones difíciles (comunidades pobres, escasos recursos, geográficamente aisladas).

Para obtener un mejoramiento continuo de la Gestión Escolar, se necesita que las Instituciones Educativas generen acciones curriculares y administrativas que permitan que diversos actores asuman un rol de liderazgo más participativo y se comprometan activamente en la tarea de lograr mayor motivación dentro de las diferentes áreas académicas (Directivos, Administrativos, Docentes, etc.), esto se puede dar mediante incentivos, capacitación, remuneraciones económicas, entre otros.

Se requieren directivos dentro de las Instituciones Educativas con un alto nivel de conocimientos profesionales, herramientas de liderazgo y de Gestión Educativa, sin embargo, en la mayoría de las ocasiones, éstos no tienen la capacidad de dirigir y mucho menos tienen participación en la selección de personal, además la mayoría de ellos, no tienen una autonomía de liderazgo siendo ésta una de las dificultades más notorias de su gestión.

En la Actualidad, el sistema educativo requiere directivos, profesores y alumnos involucrados con todas sus capacidades y valores. Ello implica el desarrollo de capacidades y competencias de liderazgo, así como la generación de ambientes propicios para una sana convivencia de todos los integrantes del sistema educacional; un ambiente democrático y participativo, donde la visión que guíe la gestión escolar sea ampliamente compartida, la línea de escuelas "efectivas", plantea, que el mejoramiento educativo y éxito escolar de los estudiantes se logra por lo tanto no solo en el salón de clases.

Es necesario desarrollar en los estudiantes habilidades funcionales como las de pensamiento (bastante difícil en ésta Era de la Tecnología) además habilidades intrínsecas, que les permitan implementar su efectividad personal y de empleabilidad; el proceso de enseñanza- aprendizaje debe ayudar al alumno a un desenvolvimiento más favorable, bajo ciertas condiciones institucionales como el compromiso docente y a la vez involucrando a todos los agentes en la toma de decisiones con un buen liderazgo dentro de la institución, una planificación colaborativa, condiciones efectivas de trabajo, investigación y reflexión.

Las nuevas modificaciones del sector educativo, sobre todo la entrada de nuevas tecnologías educacionales y el mundo globalizado en que se vive actualmente, atraen otras necesidades formativas ante las cuales el aula y la institución se ven vulnerables e impotentes al no satisfacer las necesidades actuales.

La formación inicial y las necesidades educacionales son insuficientes para satisfacer las demandas de las instituciones educativas, esta insuficiencia se da por dos razones:

- La oferta formativa está desconectada del mundo de las necesidades sociales.

- El desarrollo de la formación se apoya más en la teoría que en la práctica. 
Las instituciones educativas juegan el papel de preparar a los alumnos para ejercer una profesiòn, sin embargo, ni la curricula ni aún los profesores son capaces de orientar esos estudios a la adquisiciòn de las hàbilidades propias de la profesiòn, existe la necesidad de encontrar una adaptaciòn, por un lado, la formaciòn con el trabajo y por otro lado a la producción con la innovación.

Se podría decir que históricamente, la dirección ha estado ligada a una persona y sólo en la época más reciente se ha contemplado la formación de equipos directivos, delegando funciones y participación en el resto de la comunidad educativa. El concepto "equipo" es solamente nombrado por los directivos (entre los 15 conceptos de mayor peso semántico). Por otra parte, se habla de "jefe", lo que podría asociarse a "director" = líder.

Esto es reafirmado por Beck y Murphy (citados en López et al., 2003), los que en los años 90' destacaron la imagen del “director como líder", quien, además de las competencias asignadas oficialmente a su rol, era capaz de orientar el cambio, motivando, resolviendo conflictos, influyendo con base a su experiencia profesional y capacidades, más que a través de la autoridad, o en una gestión más moderna de un liderazgo sustentable, como lo promueve Hargreaves (2005).

Es importante recordar que no existe un solo estilo de liderazgo para todas las circunstancias. Si bien se podría considerar el "liderazgo transformacional" como el más apropiado para los requerimientos actuales de la gestión educacional, se estima necesario adecuar el rol directivo al contexto en el que deben manifestarse ciertas competencias que le permitan al líder adaptarse a los cambios futuros.

De acuerdo con Fernández (1995), se considera que una premisa importante a seguir es adaptar el estilo de liderazgo a las demandas culturales del entorno en que se trabaja.

\section{2.- EVALUACIÓN Y CALIDAD}

La evaluación y la calidad han tenido un frágil y sinuoso camino dentro del sistema de educación superior en México. Se llegó tarde frente a lo que con frecuencia se erige como un paradigma, por ejemplo: los sistemas que en ese mismo ámbito tienen establecidos Estados Unidos y Canadá.

El Tratado de Libre Comercio con los países de Estados Unidos y Canadá y la vigencia que tiene desde 1994, han permitido observar el funcionamiento de esos sistemas, parte de los planeamientos que se hicieron en los años 1993 y 1994 recomendaron hacer nuevos instrumentos de evaluación y calidad en dos capítulos del Tratado de Libre Comercio, dónde se hace mención del intercambio e ingreso de profesionales, así como la acreditación y certificación.

En 1984 hubo una propuesta por parte de las universidades para implantar los procesos de evaluación y calidad, pero por circunstancias ajenas a las instituciones fue hasta el año de 1991 cuando se dieron los primeros pasos en estas materias. 
La evaluación y la calidad en México han sido planteadas como un mecanismo para fomentar una mejora para la educación y se ha llegado a lograr una mayor calidad en los procesos y resultados además de ser una inquietud para el sistema educativo, es considerada también un atributo imprescindible porque la educación debe de ser de calidad.

La evaluación y la calidad son procesos y medios necesarios para el mejoramiento de los sistemas de educación, en el caso de México, los planteamientos en esta materia se han hecho con el debido interés para que estos puedan responder a sus propias circunstancias históricas sociales y educativas.

En la medida en que la evaluación represente ese proceso, es posible orientar las tareas educativas de la formación profesional de acuerdo con prácticas y resultados ampliamente reconocidos convirtiéndose así en un medio indispensable para el mejoramiento en la calidad, de este punto se parte para hacer notar que la evaluación tiene un papel estratégico dentro de las políticas educativas orientadas a promover los caminos relevantes de la organización con eficiencia y eficacia.

\section{Desde el informe Pisa:}

La finalidad de la evaluación será entonces examinar el grado que existe entre los planteamientos generales y los elementos del currículo (objetivos, contenidos, criterios de evaluación) más cercanos a la práctica docente en el aula, así como los distintos componentes que configuran en la competencia científica y según PISA en los objetivos generales del área, en los bloques de contenidos y en los criterios de evaluación del ciclo o curso. Sin embargo, no debe olvidarse la construcción social del conocimiento, haciendo hincapié en el carácter humano de la ciencia, para formar ciudadanos competentes ya que, en una sociedad, el acercamiento de las personas a los científicos y a su quehacer es de suma importancia para la incorporación de ese conocimiento.

La comparación entre las competencias, utilizando como referente la estructura del informe PISA, nos indica que las diferencias son relativas a los contenidos sobe la ciencia y las capacidades o destrezas necesarias para su aprendizaje y utilización de contextos ciudadanos, la preocupación por medir es ahora prioritaria globalmente.

La construcción social del conocimiento científico o el carácter humano de la ciencia, es más objetivo en la utilización de contextos. La competencia científica lleva a PISA a la acreditación de capacidades tales como:

- Identificar cuestiones científicas.

- Explicar fenómenos científicamente.

- Extraer conclusiones basadas en pruebas.

- Haber adquirido conocimientos que permitan comprender el mundo natural.

- Algunos rasgos acerca de la propia ciencia.

- Saber utilizar estos conocimientos en contextos de interés. 
- Mostrar actitudes como el interés hacia la ciencia y la investigación científica.

Nos encontramos en una época, donde las instituciones educativas, están en constante transformación, buscando estrategias que contribuyan al mejoramiento de la calidad, esto para que los alumnos cuenten con habilidades, destrezas y conocimientos que les permitan enfrentarse y ser competitivos en cualquier área productiva; para que esto sea realmente un hecho, la institución debe conocerse, contextualizarse y planear de manera permanente acciones que la lleven a cumplir los objetivos planteados.

La evaluación y la calidad, son el binomio perfecto que puede lograr que la Institución Educativa, se encamine hacia la denominada "mejora continua".

De acuerdo al PEC (Programa Escuelas de calidad.2001, 104)...."La evaluación como medio para la revisión de los procesos y resultados en el aula, en la escuela, en la zona o sector y en los niveles del sistema permite la formulación de estrategias y de acciones de intervención propias para cada situación, mismas que deben discutirse, acordarse y consensuarse para potenciar la satisfacción colectiva en función de mejoras sustanciales.

Para tal efecto, se requiere de una evaluación inicial que sirva como punto de partida y comparación respecto de las posibles mejoras, así como la evaluación de seguimiento y la final, para reconocer el alcance de las metas, como punto de llegada, en un ciclo escolar. En este sentido, cada ciclo de planeación tiene como instrumento y eje transversal a la evaluación. El ciclo de mejora puede representarse de la siguiente manera:

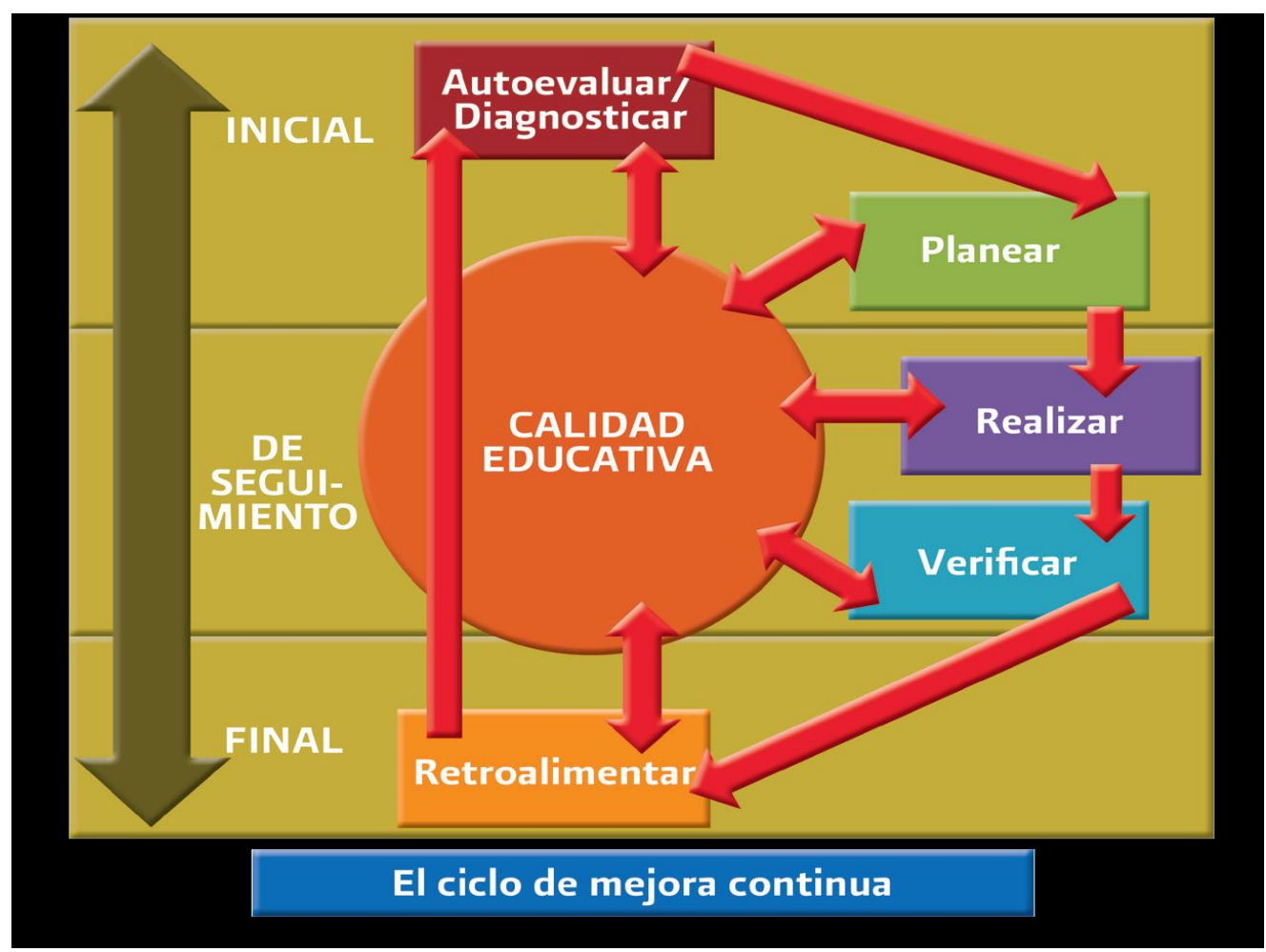

Fuente: F. Javier Murillo y Gabriela J. Krichesky. 2011 
Uno de los procesos que auxilian a la Institución Educativa a conocer las fortalezas y debilidades de la misma es la Evaluación Institucional, ya que ésta se encarga de arrojar información sobre un sinfín de situaciones, identificando y reconociendo características de las diferentes áreas como son:

- Gestión escolar.

- Finanzas.

- Investigación.

- Formación Docente.

- Mercadotecnia.

- Servicios Escolares.

- Direcciones Académicas, etc.

Lo anterior, conlleva a que los actores responsables que se encuentran dentro de la Institución, gestionen y asuman de manera dinámica las exigencias que presenta la evaluación institucional, generando una reflexión sobre las acciones que realizan, propiciando el cambio y aminorando la resistencia al mismo.

Hablar de procesos, implica hablar de evaluación institucional, la cual tiene como objetivo, mejorar la calidad de una institución a través de interrogarse sobre los resultados y especialmente las acciones, identificando problemas y comprendiéndolos en su contexto. Por tanto, esta debe servir para interpretar, cambiar y mejorar, no para normativizar, prescribir y mucho menos como una "actividad punitiva".

La evaluación institucional es un proceso continuo y con carácter constructivo, que considera tanto los aspectos cualitativos como los cuantitativos y se realizará en forma permanente y participativa (CONEAU,1997).

Tanto la Calidad como la Evaluación, representan un cambio dentro de las Instituciones Educativas, los cambios tienen implícito un gran proceso que conllevan algunas etapas importantes como la de "sensibilización", ésta, se debe dar por medio de personas capacitadas para ello como son los psicólogos educativos, realizando de antemano un diagnóstico inicial.

Este paso, estaría asegurando el éxito de cualquiera de los dos parámetros y la comunidad educativa reaccionaría con mayor aceptación a ese cambio.

Posteriormente, ya se podría hablar de la Nueva Reforma Educativa que trae consigo a la Evaluación y a la Calidad.

\section{3.- POLÍtica EDUCATIVA}

Según (Fernández, Noelia 2014 en Rizvi, F. y Lingard, B. (2013) "En un sentido amplio, consideran que los debates sobre las políticas públicas, y en lo que nos ocupa, sobre políticas educativas, se están llevando a cabo desde los postulados de una globalización 
neoliberal que rechaza el Estado keynesiano. Al hilo de este argumento vale la pena recordar la tesis que ya en 1997 sostuvo Cerny: las profundas transformaciones representadas bajo el concepto de globalización no se refieren sino al paso del "Estado keynesiano de Bienestar" al "Estado Competitivo". Esto ha afectado al rol de la educación."

Bajo este contexto neoliberal en México y después de insertarse dentro de la Globalización, surge el programa de «Mejoramiento de la Calidad en la Escuela Primaria» en dónde se focalizan las asignaturas de español y matemáticas como las de mayor relevancia, sin descuidar las demás y que actualmente se trabaja en todos los municipios y localidades del interior de la República.

Cómo parte de la estrategia se evaluó la preparación profesional, el aprovechamiento de los diversos programas en beneficio de las escuelas que participan, como por ejemplo las escuelas de Calidad o PEC.

Uno de los objetivos dentro de la línea estratégica es el fortalecimiento e impulso de los procesos de formación, capacitación, actualización y superación permanentemente de los profesionales de la educación en los diferentes tipos de niveles y modalidades educativas a fin de desarrollar sus capacidades, actitudes, habilidades y destrezas laborales.

Uno de los principales soportes de la reorganización en la administración educativa ha sido la desconcentración de los diversos trámites y servicios y su aplicación ha logrado una mejoría sustantiva de la atención de los servicios educativos al acercar la administración a las regiones, localidades y municipios para coadyuvar al logro de los bienes educativos y disminuir así las diferencias entre los diversos municipios y regiones del país.

El impulsar la participación social en el punto educativo, en el apoyo a la escuela a la labor del maestro y en beneficio del desarrollo integral del educando comprometiendo a la comunidad en la búsqueda constante de alternativas y estrategias para mejorar la calidad de la educación básica, constituye una de las principales políticas públicas en una sociedad democrática.

En complemento a lo anterior, la planeación es el elemento básico para conjuntar ideas viables, fijar objetivos, metas, estrategias y prioridades, dar coherencia a las acciones, prever los recursos, apoyos y servicios que aseguren la prestación y el desarrollo de los servicios educativos que presta el Estado.

De lo anterior se desprende, que las políticas educativas, necesitan por ende del factor planeación, y el éxito de la planeación, depende de la capacidad de un grupo u organización de exponer sus ideas sobre otros grupos y organizaciones, el ejemplo anterior nos da muestra de ello cuando un presidente habla de políticas educativas.

La calidad de las ideas puede influir en la generación y ejercicio del poder, pero no serán esas ideas en sí, la explicación del "éxito" en la implantación del plan, sino que dependerá de otros factores. 
Se considera, dice McGinn y Warwick (2006) que el éxito de la planeación, se da en función de las habilidades en el desarrollo y manejo de las relaciones políticas y no de la competencia técnica. Se considera a la planeación esencialmente como proceso político, en que se manipula información empleando técnicas con una alta carga de legitimidad científica, para imponer nuevas formas de organización.

A la vez, la planeación ha creado grandes expectativas de cambios y de esta manera, ha servido para mantener por un tiempo más, la estructura básica del sistema político y económico.

Aquí la reflexión invita a pensar realmente ¿qué ha cambiado y qué se ha logrado en nuestro País? ¿Las nuevas políticas de éste sexenio están encaminadas a resultados fehacientes?

\section{4.- REFORMA EDUCATIVA}

¿Para qué nos sirve en México? Para crear el Sistema de Servicio Profesional Docente, el cual está integrado por concursos de ingresos para docentes y para la promoción a cargos con funciones de dirección y de supervisión en la educación básica y media superior. Con dicha creación se estarían fijando los términos para el ingreso, la promoción y la permanencia en el servicio. Se forma también el Instituto Nacional para la Evaluación de la Educación (INEE) que tiene atribuciones para evaluar el desempeño y resultados del Sistema Educativo Nacional. El INEE está formado por personas con reconocida capacidad y es un cuerpo colegiado compuesto por 5 Consejeros, aunque el presidente de la junta de gobierno del INEE es propuesto ante el Senado de la República por el titular del Poder Ejecutivo nacional. La evaluación magisterial en primer lugar, no es voluntaria, sino obligatoria para todos los maestros; es decir, el maestro que repruebe la evaluación se tendrá que ir a capacitar, para concretar una nueva evaluación.

En el Diario Oficial de la Federación del día 26 de febrero de 2013 se decreta lo siguiente:

"DECRETO por el que se reforman los artículos 3o. en sus fracciones III, VII y VIII; y 73, fracción $X X V$, y se adiciona un párrafo tercero, un inciso d) al párrafo segundo de la fracción II y una fracción IX al artículo 3o. de la Constitución Política de los Estados Unidos Mexicanos".

El artículo $3^{a}$.- El Estado garantizará la calidad en la educación obligatoria de manera que los materiales y métodos educativos, la organización escolar, la infraestructura educativa y la idoneidad de los docentes y los directivos garanticen el máximo logro de aprendizaje de los educandos.

Uno de los puntos importantes que toca esta reforma educativa es la de una educación con calidad, con base en el mejoramiento constante y el máximo logro 
académico de los educandos, además de que la educación debe garantizar, la prestación de servicios educativos de calidad, se crea el Sistema Nacional de Evaluación Educativa.

La coordinación de dicho sistema estará a cargo del Instituto Nacional para la Evaluación de la Educación. El INEE será un organismo público autónomo, con personalidad jurídica y patrimonio propio. Corresponderá entonces al Instituto evaluar la calidad, el desempeño y resultados del sistema educativo nacional en la educación preescolar, primaria, secundaria y media superior, generar y difundir información y con base en estas directrices que son relevantes para contribuir a las decisiones a mejorar la calidad de la educación y su equidad, como factor esencial en la búsqueda de la igualdad social

La creación de un Sistema de Información y Gestión Educativa en efecto, fue muy importante ya que durante el año 2013 el Instituto Nacional de Estadística y Geografía realizó un censo de escuelas, maestros y alumnos, que permitió a la autoridad tener en una sola plataforma los datos necesarios para la operación del sistema educativo a su vez, una comunicación directa entre los directores de escuela y las autoridades educativas.

El uso de la evaluación del desempeño docente para dar mayor pertinencia y capacidades al sistema nacional de formación, actualización, capacitación y superación profesional para maestros, fue imperante en el marco de la creación de un servicio profesional docente. La evaluación de los maestros debe tener, como primer propósito, el que ellos y el sistema educativo cuenten con referentes bien fundamentados para la reflexión y el diálogo conducentes a una mejor práctica profesional.

El sistema educativo deberá otorga los apoyos necesarios para que los docentes puedan, prioritariamente, desarrollar sus fortalezas y superar sus debilidades. Así como fortalecer la autonomía de gestión de las escuelas ante los órdenes de gobierno que corresponda con el objetivo de mejorar su infraestructura, comprar materiales educativos, resolver problemas de operación básicos y propiciar condiciones de participación para que alumnos, maestros y padres de familia, bajo el liderazgo del director, se involucren en la resolución de los retos que cada escuela enfrenta.

\section{5.- CONCLUSIONES}

Después de haber analizado, estos cuatro ejes de intersección en la Educación en México, nos podemos dar cuenta de que la reflexión final, podría estar ligada directamente con la importancia que se le ha otorgado a cada uno de éstos ejes en nuestros días, ya que pareciera que uno no puede existir sin ir de la mano con el otro, como si se tratara de un entretejido que por supuesto afecta a nuestra sociedad en todo lo educativo.

Es decir, el liderazgo se ha convertido en parte esencial de una buena gestión, la calidad no debiera darse sin una evaluación, la política educativa se entrelaza a su vez con la nueva reforma como si no existiera ninguna por separado, esto representa el mundo actual en el que estamos inmersos día con día. 
Las instituciones educativas deben moverse ahora hacia la modernidad a pasos agigantados y tal pareciera que sin tomar en cuenta estos cuatro ejes, no podrán alcanzar sus objetivos a corto o mediano plazo ¿Qué nos espera entonces si no se les toma en cuenta? ¿Podríamos llegar al caos de no existir una cultura de evaluación en México? ¿Sabemos realmente lo que es la calidad educativa? ¿Esperamos concretar de una manera exitosa el modelo en competencias? ¿Podemos aplicar la nueva reforma educativa en toda su extensión?

Estas y otras interrogantes nos esperan a los que nos dedicamos a la educación, aunque no estamos exentos de una gran responsabilidad para que se concreten las acciones de manera efectiva en nuestras instituciones educativas, pues nos corresponde, como siempre, contribuir con nuestro granito de arena.

\section{BIBLIOGRAFIA}

MUÑOZ IZQUIERDO, Carlos. Resultados de las políticas educativas nacionales. Observatorio Ciudadano de la Educación. Foro Nacional la Educación en las Plataformas Electorales, México. 2000.

PAZ TAPIA-GUTIÉRREZ, Carmen, BECERRA-PEÑA, Sandra, MANSILlASEPÚlVEDA, Juan, SAAVEDRA-MUÑOZ, July. Liderazgo de los directivos docentes en contextos vulnerables. Educación y Educadores, vol. 14, núm. 2, mayoagosto, 2011, pp. 389-409, Universidad de La Sabana. Colombia.

Programa de Desarrollo Educativo 2003-2008.

Programa Escuelas de Calidad. Modelo de Gestión Educativa. 2a. Edición SEP. México 2010.

RIZVI, F. y LINGARD, B. Políticas Educativas en un Mundo Globalizado. Madrid: Morata. 2013.

RUIZ CALLEJA, José Manuel. Dirección y gestión educativa. Colección Medellín, Esumer, primera edición. 2004

SÁNCHEZ, Verónica, TAPIA, Carmen, BECERRA, Sandra. Liderazgo: una mirada desde directivos, profesores y alumnos. Revista de Psicología, vol. XV, núm. 2, 2006, pp. 29-52, Universidad de Chile.

SAÑUDO, Lya. La transformación de la gestión educativa. Entre el conflicto y el poder. <http://educacion.jalisco.gob.mx/16/16Lya.html>. Educar, revista de educación, nueva época, núm 16, enero-marzo. 2001 
ULLOA GARRIDO, Jorge; NAIL KRÖYER, Oscar; CASTRO HIDALGO, Abelardo; MUÑOZ REYES, Máximo. Problemas de gestión asociados al liderazgo como función directiva. Estudios Pedagógicos, vol. XXXVIII, núm. 1, 2012, pp. 121-129 Universidad Austral de Chile Valdivia, Chile. 